\title{
Experimental Studies on Improved Plastic Bonded Explosives Materials (PBXs) for Controlled Fragmentation Warheads
}

\author{
Karim Elsharkawy ${ }^{1, a}$, Lin Guo ${ }^{1}$ and Hany Fouda ${ }^{1}$ \\ ${ }^{1}$ School of Chemistry and Environment, Beijing University of Aeronautics and Astronautics, Beijing 100191, China
}

\begin{abstract}
This paper describes formulations of plastic bonded explosives (PBXs) based on three highly brisant explosives, namely 1,3,5-trinitro-1,3,5-triazinane (RDX), 1,3,5,7-tetranitro1,3,5,7-tetrazocane (HMX) and pentaerythritoltetranitrate (PETN) with polyurethane (PU) based on Glycidyl azide polymer (GAP) as an energetic binder in comparison with composition-B, which used in the fragmentation warheads. The sensitivity and performance properties of different types of PBXs were evaluated by experimental results of prepared selected PBX compositions. Casting technique was used to prepare the selected compositions containing $14 \%$ PU based on GAP. It has been observed that the brisance of the PBX based on HMX was higher than that of comp-B by $21.3 \%$, the detonation velocity showed a remarkable increase of the order of $8480(\mathrm{~m} / \mathrm{s})$ while that of comp-B was $7638(\mathrm{~m} / \mathrm{s})$. A controlled fragmentation warhead with an outer grooving warhead case of dimensions $100 \times 35 \times 4 \mathrm{~mm}$ was used and arena test was carried out to determine the lethal zone of the fragmentation warhead. The lethal zone obtained from arena test for PBX composition based on HMX named PBXHG4 was higher than that based on RDX or PETN, and than that of comp-B by $40 \%$.
\end{abstract}

\section{Introduction}

The new development in the field of explosives is to develop a new class of explosives known as plastic bonded explosives (PBXs) which have significantly lower vulnerability to various stimuli than conventional high explosives as comp-B that suffers from various disadvantages such as relatively poor impact sensitivity, cure shrinkage which results in voids and cracks, and somewhat violent reaction during cook-off [1-3]. By the end of the First World War, the explosives development takes the direction to improve the explosive performance, and as a result, the sensitivity of explosives has been increased. Over the years, many accidents were happened during handling, transporting, testing and manufacturing of explosives, where many researchers have always paid attention to increase performance without considering safety aspect. The demand for increased safety in explosives transportation, handling, and storage has led to the improvement of Insensitive Munitions (IM) [4]. The design of these weapons and explosives decreases the probability of unwanted and unexpected detonation from external stimuli such as weapon fragments, heat, and shock. This can be achieved by the modification to the explosive formulation.

Plastic bonded explosive (PBXs) are various explosive mixtures where the crystals of high explosives are embedded into a thin layer of polymeric material. Recently, many researches focused on the preparation, characterization and applications of modern of PBX formulations based on high

\footnotetext{
${ }^{a}$ Corresponding author: karimelsharkawy@buaa.edu.cn

(C) The Authors, published by EDP Sciences. This is an open access article distributed under the terms of the Creative Commons Attribution License 4.0 (http://creativecommons.org/licenses/by/4.0/).
} 
brisance explosives and energetic polymeric binders to achieve better explosive properties and low vulnerability [5-8]. Controlled fragmentation warheads are used to defeat almost all types of targets (underwater, aerial, human and underground) to set them out of action by the action of fragments created from the warhead body at the time of explosion. The velocity of fragments depends mainly on the detonation velocity or brisance of the high explosive. Higher fragment velocity and large lethal zone could be obtained by using high performance explosive material $[9,10]$.

\section{Experimental}

\subsection{Preparation of Plastic bonded explosives}

All the chemicals used in this work; RDX, HMX, PETN, GAP, and Isophoron diisocyanate (IPDI crosslinking agent) were of high purity. These PBXs formulations based on polyurethane were prepared by using the casting technique under vacuum [11]. This process was conducted in a stainless steel bowl of $5 \mathrm{~kg}$ capacity provided with double wall jacket, where a circulating liquid could be either heated or cooled. Stainless steel bowl is equipped with a vertical mixer with three blades rotating in an orbital motion. The prepolymer used in this work was GAP (1.43 measured $\mathrm{mg}$ equivalent $\mathrm{OH} / \mathrm{g}$ GAP). The prepolymer was precisely dropped into the reactor and then preheated until the temperature reaches to $60{ }^{\circ} \mathrm{C}$ then the dried pure explosive was divided into four equal portions and added during stirring without vacuum for 8 minutes for every portion. After adding the fourth portion of the explosive, the mixer was kept under vacuum for 18 minutes to avoid the presence of air bubbles in the mixing samples. Finally, the curing agent (IPDI) was added and mixing was carried out for relatively short time. The explosive mixture was then poured in polyvinyl chloride (PVC) mould tubes and experimental controlled fragmentation warhead to measure detonation velocity and lethal zone respectively after curing which was carried out at $55-60{ }^{\circ} \mathrm{C}$ for 12 days. Three PBX formulations ( $86 \%$ explosive $+14 \%$ binder) were prepared at the same binder PU based on GAP, but at different explosive filler HMX, RDX, and PETN, which designated as PBXHG4, PBXRG4, and PBXPG4 respectively.

\subsection{Evaluation of PBXs formulations}

\subsubsection{Sensitivity tests}

Sensitivity to friction was determined using BAM friction test apparatus. The frictions test was determined by the percentage of initiation by changing the loading of the pistil [12]. Sensitivity to impact was carried out using IKa Maschinenbau apparatus, using $5 \mathrm{Kg}$ falling weight [13]. Upper sensitivity limit was used to identify the minimal height at which $100 \%$ initiation was achieved. The sensitivity to heat was obtained by measuring the ignition temperature for the prepared PBX samples using Chilworth deflagration test apparatus [12]. To determine the ignition temperature, the temperature was uniformly increased $\left[5^{\circ} \mathrm{C} / \mathrm{min}\right]$ until the explosion conversion occurred.

\subsubsection{Detonation velocity}

The prepared PBX samples were pressed to a density $1.6\left(\mathrm{~g} / \mathrm{cm}^{3}\right)$ in PVC tubes of $2 \mathrm{~mm}$ inside diameter and $190 \mathrm{~mm}$ length. The Exploment -Fo-2000- Multi Channel (Swiss made) was used to measure the detonation velocity of these formulations. The time interval for a detonation wave to travel a known distance between the two fiber optic probes in microseconds was displayed with the calculated detonation velocity in $(\mathrm{m} / \mathrm{s})$ [14]. 


\subsubsection{Brisance}

This test was carried out using the brisance testing unit according to Kast technique [12], where $2.5 \mathrm{~g}$ of the explosive charge was pressed to a density of $1.6\left(\mathrm{~g} / \mathrm{cm}^{3}\right)$ into an aluminum tube of $30 \mathrm{~mm}$ height, $12 \mathrm{~mm}$ inside diameter and $4 \mathrm{~mm}$ wall thickness. Special test detonator with $0.2 \mathrm{~g}$ of lead azide and $0.4 \mathrm{~g}$ of RDX was prepared on site to initiate the explosive charge. The copper crushers used are of $9.8 \mathrm{~mm}$ height and $6 \mathrm{~mm}$ diameter. The deformation (final lengths) of copper crushers was determined after the explosion of the charges and converted into force units according to the calibration table of the copper crusher static compression force test [15].

\subsubsection{Arena test}

The arena test is used to measure the effects of high-explosive warhead detonations to determine the lethal zone. Lethal zone is one of the most important parameter of HE warhead efficiency [16]. The used controlled fragmentation warhead of dimensions $(100 \mathrm{~mm}$ length, $35 \mathrm{~mm}$ diameter and $4 \mathrm{~mm}$ thickness) made from steel 35 as shown in Fig. 1, was selected to be filled with $100 \mathrm{~g}$ of the investigated PBX formulations. In this test, we compare lethal efficiencies for similar warheads; the warhead is put on the ground at which the warhead nose is in the down position in the center of the test arena. The target is made of metallic witness panels of dimensions ( $2 \mathrm{~m}$ height, $1 \mathrm{~m}$ width and 2 $\mathrm{mm}$ thickness) placed at specified distances $(10,15,20,25,30$ and $35 \mathrm{~m})$ from tested warhead. Electric detonator was used to initiate the high explosive charge, after the detonation of the warhead, number of perforations at metallic witness sectors are counted per square meter for each sector. A characteristic distance is the fragments concentration density of one perforation per square meter. This distance is named warhead efficiency radius, which means that warhead with greater lethal zone has corresponding greater efficiency radius $[17,18]$.

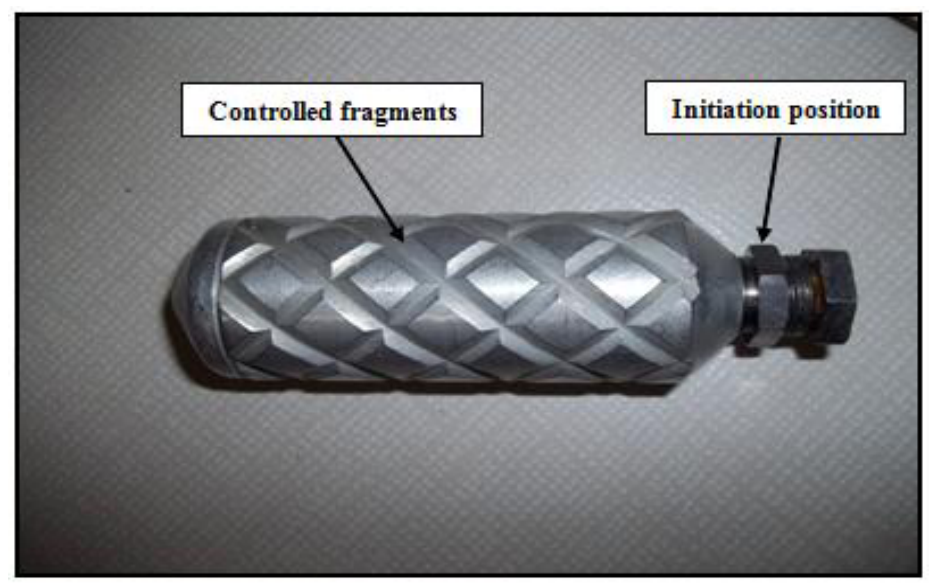

Figure 1. Photograph of the used controlled fragmentation testing cylinder casing.

\section{Results and Discussion}

\subsection{Sensitivity tests}

For comp-B $(60 \% \mathrm{RDX}+40 \% \mathrm{TNT})$, and the prepared PBX formulations, the sensitivity results are listed in Table 1. It is clear that for PBXHG4, the decrease of sensitivity to impact was $42.5 \%$ 
compared with comp-B. PBXRG4 and PBXHG4 showed no initiation even when applying the maximum friction force $(360 \mathrm{~N})$ of the test apparatus but PBXPG4 showed initiation because of PETN is high sensitive to friction. PBXs showed ignition temperature slightly lower than comp-B. This can be attributed to the fact that the coating of polyurethane based on GAP acts as heat sensitizing medium because its softening temperature is less than $152{ }^{\circ} \mathrm{C}$. PBXHG4 has sensitivity to heat close to that of comp-B. The values of ignition temperature for the prepared PBX formulations and comp-B are listed in Table 1.

\subsection{Detonation velocity}

The detonation velocity of explosives is one of the important terms which represent the rate of energy delivery through explosive conversion. From the obtained results we can find that the type and content of binder have a significant effect on detonation velocity of the prepared PBX formulations. The values of detonation velocity (D) were determined for comp-B and the prepared PBX formulations are listed in Table 1 . For PBXRG4, detonation velocity increased by $6.9 \%$ but increased by only $3 \%$ for PBXPG4. For PBXHG4, the increase of detonation velocity was $11 \%$, when compared with that of comp-B.

\subsection{Brisance}

The values of brisance (B) were determined for comp-B, and the prepared PBX formulations are listed in Table 1. Brisance were slightly increased for PBXPG4 by $6.9 \%$ and for PBXRG4 increased by $9.2 \%$, for PBXHG4 the increase of brisance was $21.3 \%$, when compared with that of comp-B, which mean a great improvement in shuttering effect, improved velocity of the fragment and the lethal zone.

Table 1. Sensitivity and performance results of comp-B and PBX formulations based on PU (GAP).

\begin{tabular}{|c|c|c|c|c|c|}
\hline Explosive & Impact (J) & Friction (N) & $\begin{array}{c}\text { Ignition } \\
\text { temp. }\left({ }^{\circ} \mathbf{C}\right)\end{array}$ & $\begin{array}{c}\text { Detonation } \\
\text { velocity (m/s) }\end{array}$ & $\begin{array}{c}\text { Brisance } \\
(\mathbf{k p})\end{array}$ \\
\hline Comp-B & 12.0 & 220 & 239 & 7638 & 1059 \\
\hline PBXRG4 & 19.8 & $>360$ & 232 & 8165 & 1157 \\
\hline PBXHG4 & 17.1 & $>360$ & 234 & 8480 & 1285 \\
\hline PBXPG4 & 15.3 & 238 & 228 & 7867 & 1132 \\
\hline
\end{tabular}

\subsection{Arena test}

The lethal zone is one of the most important parameters of high explosive warhead efficiency. The warhead lethality zone for comp-B and the prepared PBX formulations are shown in Fig. 2. Only one fragment is sufficient to kill or affect on the target so, a horizontal line was sketched at fragment density $=1$ and the radius of lethality zone for each examined explosive charge was obtained from the $\mathrm{X}$ axis. It is clear that warhead filled with PBXHG4 has a greater lethal efficiency $(28 \mathrm{~m})$ than PBXRG4 (25 m), PBXPG4 (23 m) and comp-B (20 m). For PBXHG4 the increase of efficiency radius was $40 \%$, when compared with that of comp-B. From Fig. 3, it is clear that fragments sizes were 
controlled as regular and effective from warhead casing to avoid its random distribution which could be achieved by grooving the outer surfaces of the warhead casing. After the explosion of the warhead; the explosion force affect on the weak positions in the warhead structures which are located between the formed fragments and produce a controlled fragment size and shape.

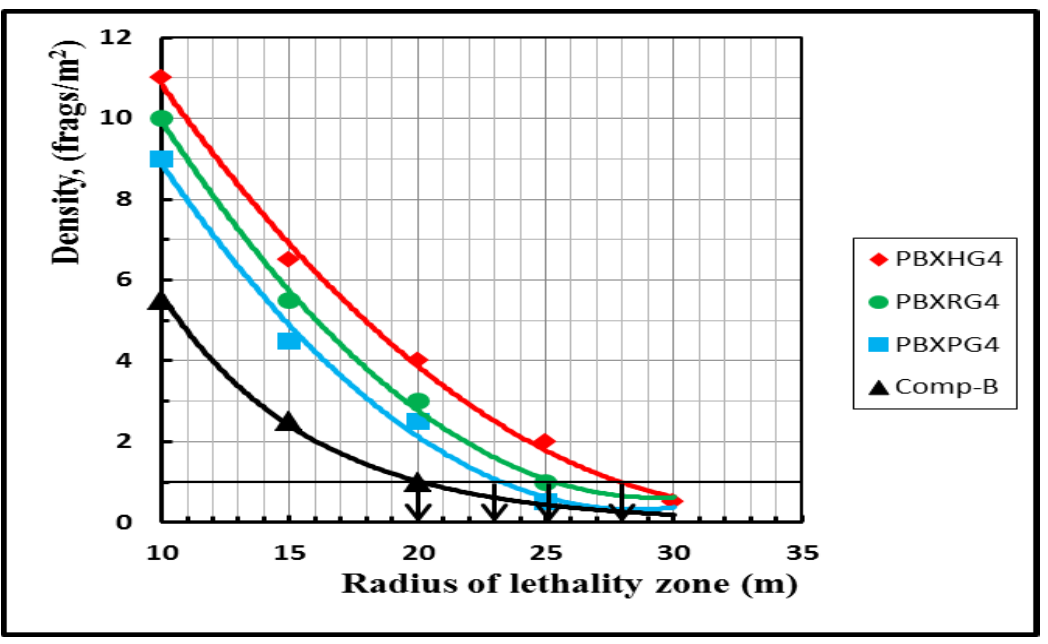

Figure 2. Warhead lethality zone of the warhead filled with comp-B and PBX formulations.

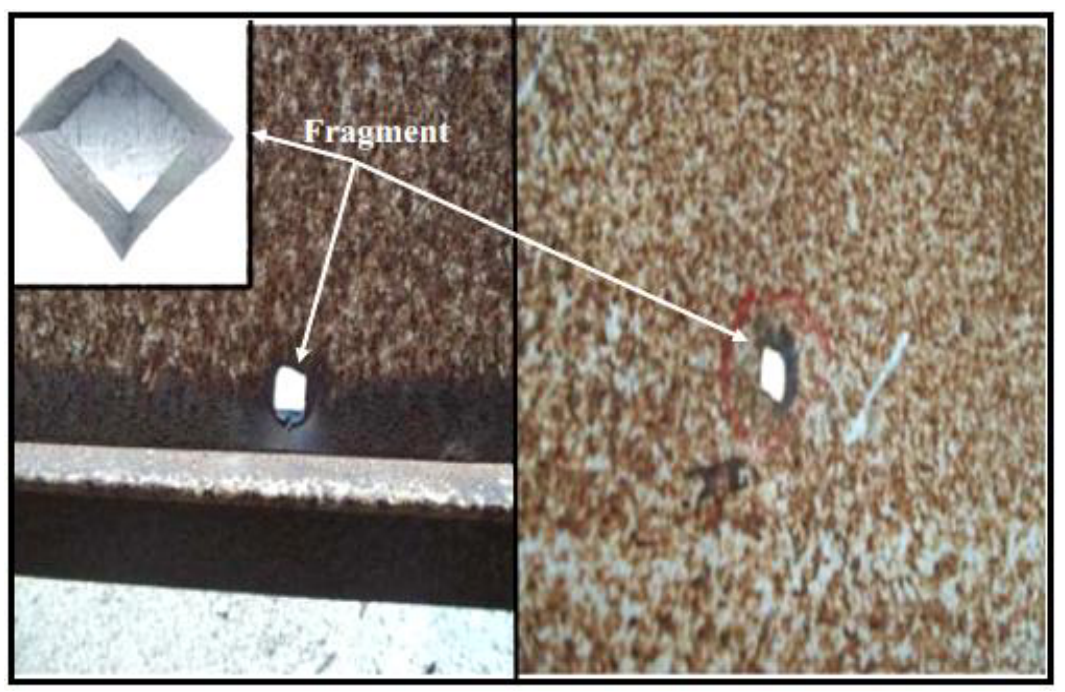

Figure 3. Photograph of the controlled fragment after the detonation of the warhead and its effect on the witness sectors.

\section{Conclusions}

In the present study, three PBX formulations based on $86 \%$ explosive material (RDX, HMX, and PETN) and $14 \%$ binder (PU based on GAP) have been formulated using casting technique and investigated for explosive and performance properties. The choice of this technique increases the safety during production. The measured sensitivity characteristics proved that PBXHG4 has lower 
sensitivity to the mechanical stimulus (impact, friction) than that of comp-B by $42.5 \%$ for impact sensitivity. Its sensitivity to heat is close to that of comp-B. PBXHG4 has higher performance parameters, in which detonation velocity increased by $11 \%$, brisance increased by $21.3 \%$ and lethal zone increased by $40 \%$ when compared with that of comp-B. PBXs possess several advantages: ease and simplicity of production, lower sensitivity to impact and friction, suitable heat sensitivity, performance and increased safety. Thus, it can be said that these formulations especially PBXHG4 which have high values of brisance, are recommended to be used as a main explosive charge of low sensitive fragmentation warheads and demolition charges instead of composition-B.

\section{References}

1. T.Urbanski, "Chemistry and Technology of explosives" Pergamon press, New York, USA, 4 (1985)

2. Doll D. W., Hanks J. M., Niles J. H., "Insensitive Composition B Replacement" Insensitive Munitions and Energetic Materials Technology Symposium, Tampa, Florida, USA, 2, 264 (1999)

3. M. M. Ahmed, M. H. Abuuznien, B. M. Sidig, " Replacement of Comp-B by Plastic Bonded Explosives In a Selected Warhead" $7^{\text {th }}$ International Conference on Chemical \& Environmental Engineering, Cairo, Egypt, (2014)

4. A. Singh, M. Kumar, P. Soni, M. Singh, A. Srivastava, " Mechanical and Explosive Properties of Plastic Bonded Explosives Based on Mixture of HMX and TATB" Defence Science Journal, 6, 622-629 (2013)

5. M. Radwan, "Explosive Characteristics of Aluminized PBXs Based on HMX and Polyurethane Binder", $32^{\text {nd }}$ International Annual Conference of ICT, Karlsruhe, Germany (2001)

6. I.Plaksin, J.Campos, R.Mendes "Shock sensitivity and detonation performance of PBX formulations based on the RS-RDX, RS-HMX and fine/ultra-fine HE particles bonded with the inert binders" $39^{\text {th }}$ International Annual Conference of ICT, Karlsruhe, Germany (2008)

7. M. H. Hamad, H. E. Mostafa, " Study of Advanced Plastic Bonded Explosives" $7^{\text {th }}$ International Conference on Chemical \& Environmental Engineering, Cairo, Egypt, (2014)

8. Q. L. Yan, S. Zeman, A. Elbeih, A. Zbynek, " The Influence of the Semtex Matrix on the Thermal Behavior and Decomposition Kinetics of Cyclic Nitramines" Central European Journal of Energetic Materials, 10, 509-528 (2013)

9. M. T. Shahraini, "Casing toughness effect on anti-air fragmentation warhead performance", $23^{\text {rd }}$ International Symposium on Ballistics, Tarragona, Spain (2007)

10. S.Waggener, "The evolution of air target warheads", $23^{\text {rd }}$ International symposium on ballistics, Tarragona, Spain (2007)

11. M. B. Talawar, S. K. Jangid, T. Nath, R. K. Sinha, S. N. Asthana" New directions in the science and technology of advanced sheet explosive formulations and the key energetic materials used in the processing of sheet explosives: Emerging trends" Central European Journal of Energetic Materials, 300, 509-528 (2015)

12. M. Suceska, "Test methods for explosives", Springer, ISBN 0-387-94555-5 (1995)

13. "Recommendations on the transportation of dangerous goods, manual of tests and criteria", Third edition, United Nations, New York and Geneva (1999)

14. "Exploment - Fo - 2000 apparatus catalouge", Switzerland (2000)

15. "OZM Research brisance apparatus catalogue", Czech Republic (2010)

16. B.Zecevic, A.Catovic, J.Terzic,"Comparison of lethal zone characteristics of several natural fragmenting warheads", Central European Journal of Energetic Materials, 5, 67-81(2008)

17. B.Zecevic, J.Terzic, A.Catovic, S.S.Kadic, "Influencing parameters on HE projectiles with natural fragmentation", $9^{\text {th }}$ New Trends in Research of Energetic Materials Seminar, Czech Republic (2006)

18. B.Zecevic, J.Terzic, F. Razic, S.S.Kadic, "Lethal Influence Factors of Natural and Performed Fragmentation Projectiles", DAAAM International Scientific Book, ISBN 978-3-902734-05-1, 219-234 (2015) 